\title{
HIGH RESOLUTION INTERFEROMETRIC OBSERVATIONS OF ECLIPSING BINARY STARS
}

\author{
XIAOPEI PAN \\ California Institute of Technology, Pasadena, CA 91125 \\ and \\ MICHAEL SHAO and M. MARK COLAVITA \\ Jet Propulsion Laboratory, Pasadena, CA 91109
}

June 10,1993

\section{Introduction}

Eclipsing binary stars are of great importance in astronomy. Now, high angular resolution measurements of eclipsing stars with the Mark III Stellar Interferometer (Shao 1988) can determine not only all of the orbital parameters, but also the luminosities and color indices of the two components, and can yield precise information for the study of the stellar evolution models. Particularly, the combination of interferometric and photometric results provide a direct measurement of stellar effective temperature. In the case where the primary component can be resolved, the results from the optical interferometer then provide a reliable determination of the absolute system parameters. In addition, eclipsing binary stars are good candidates for comparison of different techniques, i.e., interferometric, photometric, astrometric, and spectroscopic, and for checking whether systematic uncertainties exist with certain techniques.

\section{Observation and Analysis}

Observation of eclipsing binary stars is a continuation of our binary research program which started in 1988. In order to reach high resolution and good u-v coverage, we selected the baseline as long as possible, typically, $28 \mathrm{~m}$ or $32 \mathrm{~m}$. In order to improve the model fitting precisions of the fringe visibilities, 15 to 30 scans ( $75 \mathrm{~s}$ fringe tracking) per night were obtained for each candidate binary stars since automatic star searching and fringe tracking can have 150 to 200 scans each night. Three wavelengths at $800 \mathrm{~nm}, 550 \mathrm{~nm}, 500 \mathrm{~nm}$, respectively, have been used for the fringe visibility measurements simultaneously, which helped to solve the problems of multi-solutions for a limited sampling in a night. The bandwidths are $22 \mathrm{~nm}$, $25 \mathrm{~nm}$ and $25 \mathrm{~nm}$, respectively for those three channels. The dark count and sky background are measured after each scan for 5 seconds. Selected reference stars with diameter equal or less than 1 mas are observed periodically for the normalization of fringe visibility.

In the data processing detected photons in each $4 \mathrm{~ms}$ frame are integrated, and dark count and sky background are corrected, and then are averaged to $0.5 \mathrm{~s}$ visibility. A nonlinear model fitting was taken for the normalized fringe visibilities, and the separations, the intensity ratio and the diameter of the primary are determined 
for each star each night assuming the relative movement of two components of a binary star negligible during the period of measurement. The details of observation and data analysis method are referred to the paper (Pan, 1992).

\section{Preliminary results}

With the Mark III Interferometer five eclipsing binary stars have been observed so far. The basic information of these stars are listed in Table 1.

Table1. Resolved Eclipsing Binary Stars with the Mark III Interferometer

\begin{tabular}{rlrlrlrlll}
\hline HR & Name & $P^{d}$ & $e$ & $i^{\circ}$ & $a_{a . u .}$ & $d_{p c}$ & $\Delta m$ & Spectrum \\
& & & & & & & & & \\
2088 & $\beta$ Aur & 4 & 0.00 & 76 & 0.08 & 17 & 0.0 & A2IV & A2IV \\
5793 & $\alpha$ CrB & 17 & 0.37 & 88 & 0.2 & 26 & 3.8 & B9.5IV & G5 \\
936 & $\beta$ Per & 680 & 0.23 & 84 & 2.7 & 27 & 2.9 & B8V, K4IV, F0V \\
1612 & $\zeta$ Aur & 972 & 0.41 & 94 & 4.6 & 259 & 2.5 & K4I & B6V \\
1605 & $\epsilon$ Aur & 9890 & 0.20 & $\approx 75$ & $<20$ & $>500$ & 2.2 & F0I & B \\
& & & & & & & & & \\
\hline
\end{tabular}

The well-known triple system, Algol, have been observed for 5 years with the Mark III Interferometer. For the first time, the observations of fringe visibility between the central pair and the third body were made while the central pair was eclipsing. The magnitude of the eclipsing pair changed $0 .^{m} 8$ at $800 \mathrm{~nm}$ and $1 .^{m} 0$ at $550 \mathrm{~nm}$. The wide pair of Algol has had its orbit and distance determined interferometrically, which is critical to link the optical and radio reference frames. The inclinations of wide pair and close pair are close to the range of the measurement error. The third star's contribution to the system has been solved, and then will provide good input parameters for the modeling of this complicated system.

The resolution of the eclipsing binary $\alpha \mathrm{CrB}$ with the Mark III yields the inclination of $88 .^{\circ} 6 \pm 0 .{ }^{\circ} 9$ while the results from photometry is $88 .^{\circ} 2 \pm 0 .{ }^{\circ} 1$. The estimated $4 \mathrm{mag}$ difference from photometry and spectroscopy is close to $3.8 \mathrm{mag}$ at $800 \mathrm{~nm}$ from the Mark III, and confirms again the capability of resolving four magnitude differences of a binary star with interferometry.

$\zeta$ Aur is an interesting example of an atmospheric-eclipsing binary. In this system a small early-type dwarf is eclipsed by the atmospheric layers of a late supergiant, and provides a unique probe to investigate the chromosphere of a star. Observations of this system with the Mark III interferometer have resolved both the orbit and the primary. The diameter of the primary is estimated as $\sim 5$ mas at $800 \mathrm{~nm}$, and the semimajor axis as $\sim 18$ mas. The distance to this system is directly determined as $\sim 259 \mathrm{pc}$.

$\beta$ Aur is a 3.96 day period eclipsing binary with almost equal brightness of two components. The inclination from the light curve is $77 .{ }^{\circ} 76 \pm 0 .{ }^{\circ} 18$ while the value 
from the Mark III is $76 .^{\circ} 16 \pm 2 .^{\circ} 68$. This star is a member of a super cluster including the Ursa Major cluster.

$\epsilon$ Aur is a enigmatic binary system, which has no sign of the secondary in spectroscopy. But with the Mark III Interferometer the secondary has been detected. The separations is less than 2 mas, and $\Delta m$ is about 2.2 mag. This star is more than 500 pc away, and has a period of 27.1 years. We hope we won't have to wait so long to solve this low mass system.

\section{Acknowledgements}

Thanks to the people of the interferometric group at NRL and USNO for the observation time, and to Lu Rarogiewicz for assistance in observation. Literature survey is based on the SIMBAD data retrieval system, data base of the Strasbourg, France, Astronomical Data Center.

\section{References}

Pan, X. P., Shao, M., Colavita, M. M., Armstrong, J. T., Mozurkewich, D., Vivekanand, M., Denison, C. S., Simon, R. S., and Johnston, K. J. 1992, Ap. J., 384, 624.

Shao, M., Colavita, M. M., Hines, B. E., Staelin, D. H., Hutter, D. J., Johnston, K. J., Mozurkewich, D., Simon, R. S., Hershey, J. L., Hughes, J. A. and Kaplan, G. H. 1988, Astron. Astrophys., 193, 357 . 